\title{
Searching for Leptoquarks in electron-photon Collisions
}

\author{
O. J. P. Éboli ${ }^{a}$, E. M. Gregores ${ }^{b}$, M. B. Magro ${ }^{a}$, \\ P. G. Mercadante ${ }^{a}$ and S. F. Novaes ${ }^{b}$ \\ ${ }^{a}$ Instituto de Física, Universidade de São Paulo \\ C.P. 20516, 01498-970 São Paulo, Brazil \\ ${ }^{b}$ Instituto de Física Teórica, Universidade Estadual Paulista \\ Rua Pamplona 145, 01405-900 São Paulo, Brazil
}

(September 23, 2018)

\begin{abstract}
We study the production of composite scalar leptoquarks in $e \gamma$ colliders, and we show that an $e^{+} e^{-}$machine operating in its $e \gamma$ mode is the best way to look for these particles in $e^{+} e^{-}$collisions, due to the hadronic content of the photon.
\end{abstract}

Submitted to Phys. Lett. B 
The reason for the observed pattern of the fermionic generations is not elucidated in the framework of the $S U(3)_{C} \otimes S U(2)_{L} \otimes U(1)_{Y}$ Model. Nevertheless, there are theories, beyond the Standard Model that suggest a natural explanation for the existence of lepton and quark families, invoking a deeper structure which is common to both kind of fermions. Some composite models [回], for instance, suggest a preonic sub-structure, where quarks and leptons have some common constituents. This class of models exhibits a very rich spectrum which includes excited states of the known particles as well as new particles possessing unusual quantum numbers. Some of these models predict the existence of leptoquarks, which are color triplets and carry simultaneously leptonic and baryonic number. These particles are also present in several other theories beyond the Standard Model such as technicolor models [2], grand unified theories [3], and superstring-inspired models [4].

The production and signals of leptoquarks have already been analyzed in the literature for ep [5 7], hadronic [8], and $e^{+} e^{-}$colliders [9, 10]. The study of single leptoquark production in er collisions was carried out in Ref. [10] without considering the resolved photon contribution. In this work, we study the capability of an $e \gamma$ collider to search for these particles, taking into account the hadronic content of the photon. We demonstrate that the next generation of linear $e^{+} e^{-}$colliders, operating in the $e \gamma$ mode, is the best place to look for these particles in such colliders. An $e \gamma$ machine, due to the quark and gluon distributions of the photon, is able to exhibit a very rich initial state which allows a detailed study of these particles.

We shall consider an $e \gamma$ collider where the hard photons are obtained by the laser backscattering mechanism, which converts an $e$ beam into a $\gamma$ one [11,12]. The basic idea is to scatter soft photons of a few eV laser from one of the energetic beams of an $e^{+} e^{-}$linear collider. This mechanism is able to produce a collimated and energetic photon beam which 
has nearly the same luminosity and energy of the parent electron beam.

The production of leptoquarks in e collisions can occur either by direct or "resolved" photon processes, i.e. with the photon interacting through its hadronic content. In the latter case it is possible, for instance, to produce resonant leptoquarks through the quark component of the photon. We shall study the following signals for the leptoquark $S$

$$
\begin{gathered}
e+q_{\gamma} \rightarrow e+\text { jet }, \\
e+q_{\gamma} \rightarrow \text { jet }+\mathrm{E}_{\text {missing }}, \\
e+\gamma\left(g_{\gamma}\right) \rightarrow S+\text { jet },
\end{gathered}
$$

where $q_{\gamma}$ and $g_{\gamma}$ denotes the quark and gluon content of the photon respectively. The processes (11) and (2) contain the production of a leptoquark in the $s$ channel, while the process (3) is the associate production of a leptoquark and a quark jet.

For the sake of definiteness, we shall consider scalar leptoquarks predicted by the AbbottFarhi model [1], which is a confining version of the Standard Model and it is also called Strongly Coupled Standard Model (SCSM). The SCSM is described by a Lagrangian which has the same general structure of the Standard Model one. Nevertheless, no spontaneous symmetry breaking occurs and the $S U(2)_{L}$ gauge interaction is confining. In this model, the physical left-handed fermions are bound states of a preonic scalar and a preonic dynamical left-handed fermion, while the vector bosons are $\mathrm{P}$-wave bound states of the scalar preons.

The SCSM model cannot be analyzed perturbatively since it is strongly interacting in the energy scale of interest. Therefore, we shall parametrize the interaction between leptoquarks and physical left-handed fermions by an effective Lagrangian [5] given by 


$$
\mathcal{L}=\frac{\lambda^{a b}}{2}\left(S_{a b}^{\dagger} L_{a}^{T} C \tau_{2} L_{b}+\text { h.c. }\right)
$$

where $S_{a b}$ are the scalar leptoquark fields, $L_{a}$ are the physical left-handed lepton and quark $\mathrm{SU}(2)$ doublets $(a, b=1, \cdots, 12), C$ is the charge conjugation matrix, and $\lambda^{a b}$ are dimensionless coupling constants. Assuming that the leptoquark couples only with fermions of a given generation, in $e \gamma$ collisions, we can restrict ourselves to the sector of the Lagrangian that describes the leptoquark couplings to $\left(\nu_{e}, e\right)_{L}$ and $(u, d)_{L}$, i.e.

$$
\mathcal{L}=\lambda i S^{\dagger}\left(\bar{e}_{L}^{c} u_{L}-\bar{\nu}_{L}^{c} d_{L}\right)+\text { h.c. } .
$$

This Lagrangian is a prototype of a wide class of models [7] presenting the interaction of color triplet, charged scalars with $B$ and $L$ numbers, which is diagonal in the generation. There are few constraints from low energy phenomenology on models when leptoquarks are generational diagonal and the couplings to fermions are chiral [13]. Recent experimental results from $\mathrm{CDF}$ [14] were able to establish a lower bound of $M_{S}>116 \mathrm{GeV}$, assuming the branching ratio $B R(S \rightarrow e q)=1$.

The total cross section for leptoquark production in an $e \gamma$ collider can be obtained by folding the elementary cross section for the processes leading to the signals (1-3) with the electron-parton luminosity.

$$
\sigma_{e^{+} e^{-} \rightarrow e \gamma(p) \rightarrow X}(s)=\int_{z_{\min }}^{1} d z \frac{d \mathcal{L}_{e p}}{d z} \hat{\sigma}_{e \gamma(p) \rightarrow X}(\hat{s})
$$

where $z^{2}=\tau=\hat{s} / s$, with $s(\hat{s})$ being the center-of-mass energy squared of the $e^{+} e^{-}$(electronparton) system, and $d \mathcal{L}_{e p} / d z$ stands for the electron-parton $(p=\gamma, q, g)$ differential luminosity.

For direct photon processes, the $e \gamma$ luminosity is 


$$
\frac{d \mathcal{L}_{e \gamma}}{d z}=2 z F_{\gamma / e}\left(z^{2}\right)
$$

where the energy spectrum of backscattered laser photons $\left(F_{\gamma / e}\right)$ is 12

$$
F_{\gamma / e}(x)=\frac{1}{D(\xi)}\left[1-x+\frac{1}{1-x}-\frac{4 x}{\xi(1-x)}+\frac{4 x^{2}}{\xi^{2}(1-x)^{2}}\right]
$$

with $x$ being the fraction of the electron energy carried by the photon, and

$$
D(\xi)=\left(1-\frac{4}{\xi}-\frac{8}{\xi^{2}}\right) \ln (1+\xi)+\frac{1}{2}+\frac{8}{\xi}-\frac{1}{2(1+\xi)^{2}},
$$

with $\xi \simeq 2 \sqrt{s} \omega_{0} / m^{2}$, where $\omega_{0}$ is the laser photon energy, and $m$ the electron mass. In (7) we have assumed that the average number of high energy converted photons per electron is equal to one. The fraction of photons with energy close to the maximum value grows with $E$ and $\omega_{0}$. Nevertheless, $\omega_{0}$ is constrained since the laser energy should not exceed the threshold for $e^{+} e^{-}$pair creation via the interaction of the backscattered and laser photons, otherwise the conversion $e \rightarrow \gamma$ would be reduced. We have taken into account this constraint in our numerical evaluations.

Interactions initiated by "resolved" photons are described in terms of structure function of partons (quarks and gluons) inside the photon [15]. We can define the electron-parton luminosity by folding the photon structure functions with the photon distribution in the electrons,

$$
\frac{d \mathcal{L}_{e p}}{d z}=2 z \int_{z^{2}}^{1} \frac{d x}{x} F_{\gamma / e}(x) P_{\gamma}\left(z^{2} / x, Q^{2}\right)
$$

where $P_{\gamma}=Q_{\gamma}\left(G_{\gamma}\right)$ is the quark (gluon) structure function. There are several parametrizations for these structure functions available in the literature [16 18. We present our results for the fitting of Drees-Grassie (DG) [17] and we checked that they do not change in a significant way if we use, for instance, the set from Levy-Abramowicz-Charchula (LAC3) [18], 
which is characterized by a harder gluon spectrum. We evaluated all the structure functions and the strong coupling constant $\left(\alpha_{s}\right)$ at a scale $Q^{2}=\hat{s} / 4$.

Let us start our analyses by the signal of an electron accompanied by a jet. Taking into account the couplings of Eq.(5), this signal is associated to the subprocess $e q(\bar{q}) \rightarrow e q(\bar{q})$ with $q=u, d$. In the case of $e u \rightarrow e u$, there are contributions from the exchange of the leptoquark $S$ in the s-channel, and from $\gamma$ and $Z$ in the t-channel [19], leading to

$$
\begin{aligned}
\left(\frac{d \hat{\sigma}}{d \hat{t}}\right)_{e u \rightarrow e u}= & \frac{1}{16 \pi}\left\{\left(\frac{-2 e^{2}}{3 \hat{t}}+\frac{R_{e} R_{u}}{\hat{t}-M_{Z}^{2}}\right)^{2}+\left(\frac{\lambda^{2}}{2} \frac{M_{S} \Gamma_{S}}{\left(\hat{s}-M_{S}^{2}\right)^{2}+M_{S}^{2} \Gamma_{S}^{2}}\right)^{2}\right. \\
& +\left(\frac{-2 e^{2}}{3 \hat{t}}+\frac{L_{e} L_{u}}{\hat{t}-M_{Z}^{2}}-\frac{\lambda^{2}}{2} \frac{\hat{s}-M_{S}^{2}}{\left(\hat{s}-M_{S}^{2}\right)^{2}+M_{S}^{2} \Gamma_{S}^{2}}\right)^{2} \\
& \left.+\frac{\hat{u}^{2}}{\hat{s}^{2}}\left[\left(\frac{-2 e^{2}}{3 \hat{t}}+\frac{R_{e} L_{u}}{\hat{t}-M_{Z}^{2}}\right)^{2}+\left(\frac{-2 e^{2}}{3 \hat{t}}+\frac{L_{e} R_{u}}{\hat{t}-M_{Z}^{2}}\right)^{2}\right]\right\},
\end{aligned}
$$

where,

$$
L_{f}=\frac{e}{\sin \theta_{W} \cos \theta_{W}}\left(T_{f}^{3}-Q_{f} \sin ^{2} \theta_{W}\right), \quad R_{f}=-e Q_{f} \tan \theta_{W},
$$

with $Q_{f}$ and $T_{f}^{3}$ being the fermion charge in units of the proton charge, and the third component of the weak-isospin, respectively. The subprocess $e \bar{u} \rightarrow e \bar{u}$ receives contributions from the exchange of $\gamma$ and $Z$ in the t-channel and $S$ in the u-channel, resulting

$$
\begin{gathered}
\left(\frac{d \hat{\sigma}}{d \hat{t}}\right)_{e \bar{u} \rightarrow e \bar{u}}=\frac{1}{16 \pi}\left\{\left(\frac{-2 e^{2}}{3 \hat{t}}+\frac{R_{e} L_{u}}{\hat{t}-M_{Z}^{2}}\right)^{2}+\left(\frac{-2 e^{2}}{3 \hat{t}}+\frac{L_{e} R_{u}}{\hat{t}-M_{Z}^{2}}\right)^{2}\right. \\
\left.+\frac{\hat{u}^{2}}{\hat{s}^{2}}\left[\left(\frac{-2 e^{2}}{3 \hat{t}}+\frac{R_{e} R_{u}}{\hat{t}-M_{Z}^{2}}\right)^{2}+\left(\frac{-2 e^{2}}{3 \hat{t}}+\frac{L_{e} L_{u}}{\hat{t}-M_{Z}^{2}}-\frac{\lambda^{2}}{2\left(\hat{u}-M_{S}^{2}\right)}\right)^{2}\right]\right\} .
\end{gathered}
$$

The subprocesses $e d(\bar{d}) \rightarrow e d(\bar{d})$ does not involve the leptoquark $S$, however it should be included in the evaluation of the electron plus jet signal, since it is an irreducible background for this process. 


$$
\begin{aligned}
\left(\frac{d \hat{\sigma}}{d \hat{t}}\right)_{e d \rightarrow e d}= & \frac{1}{16 \pi}\left\{\left(\frac{e^{2}}{3 \hat{t}}+\frac{R_{e} R_{d}}{\hat{t}-M_{Z}^{2}}\right)^{2}+\left(\frac{e^{2}}{3 \hat{t}}+\frac{L_{e} L_{d}}{\hat{t}-M_{Z}^{2}}\right)^{2}\right. \\
& \left.+\frac{\hat{u}^{2}}{\hat{s}^{2}}\left[\left(\frac{e^{2}}{3 \hat{t}}+\frac{R_{e} L_{d}}{\hat{t}-M_{Z}^{2}}\right)^{2}+\left(\frac{e^{2}}{3 \hat{t}}+\frac{L_{e} R_{d}}{\hat{t}-M_{Z}^{2}}\right)^{2}\right]\right\}
\end{aligned}
$$

The result for $e \bar{d} \rightarrow e \bar{d}$ can be obtained from Eq. (14) by the interchange $L_{d} \leftrightarrow R_{d}$.

In Figs. 11 and 2 we show the $e$-jet invariant mass distribution for the process $e \gamma \rightarrow$ $e+$ jet, for $\sqrt{s}=500$ and $1000 \mathrm{GeV}$ respectively. We assumed $M_{S}=250 \mathrm{GeV}$ and we plotted the result for different values of $\lambda$, in particular, the choice $\lambda=0$ corresponds to the Standard Model prediction. Since the planned machines will have a luminosity of the order of $10^{4} \mathrm{pb}^{-1}$ per year, we can learn from these figures that it will be an easy task to discover the leptoquark in this mode, since there is a very clear peak well above the Standard Model background. Moreover we will be able to obtain a very precise measurement of $M_{S}$ and $\lambda$ due to the high statistic of the planned machines.

The signal (2) can be obtained through the subprocess $e u \rightarrow \nu_{e} d$ with contributions of $W$ boson in the t-channel and of the leptoquark $S$ in the s-channel, yielding

$$
\begin{aligned}
\left(\frac{d \hat{\sigma}}{d \hat{t}}\right)_{e u \rightarrow \nu_{e} d}= & \frac{1}{64 \pi}\left[\left(\frac{g^{2}}{\hat{t}-M_{W}^{2}}+\frac{\lambda^{2}\left(\hat{s}-M_{S}^{2}\right)}{\left(\hat{s}-M_{S}^{2}\right)^{2}+M_{S}^{2} \Gamma_{S}^{2}}\right)^{2}\right. \\
& \left.+\left(\frac{\lambda^{2} M_{S} \Gamma_{S}}{\left(\hat{s}-M_{S}^{2}\right)^{2}+M_{S}^{2} \Gamma_{S}^{2}}\right)^{2}\right] .
\end{aligned}
$$

We must also consider the subprocess $e \bar{d} \rightarrow \nu_{e} \bar{u}$ that exhibits contributions of $W$ in the t-channel and $S$ in the u-channel, resulting in

$$
\left(\frac{d \hat{\sigma}}{d \hat{t}}\right)_{e \bar{d} \rightarrow \nu_{e} \bar{u}}=\frac{1}{64 \pi} \frac{\hat{u}^{2}}{\hat{s}^{2}}\left(\frac{g^{2}}{\hat{t}-M_{W}^{2}}+\frac{\lambda^{2}}{\hat{u}-M_{S}^{2}}\right)^{2} .
$$

We exhibit in Figs. 3 and 4 the transverse momentum distribution of the quark (jet) for the process $e \gamma \rightarrow$ jet $+\mathrm{E}_{\text {missing }}$ for $\sqrt{s}=500$ and $1000 \mathrm{GeV}$ respectively. This distribution 
is shown for the Standard Model (i.e. $\lambda=0$ ), $\lambda=e$, and 1, assuming $M_{S}=250 \mathrm{GeV}$. Here again is quite evident the presence of the leptoquark, and due to the high luminosity expected for the e $\gamma$ machines, we should be able to measure $\lambda$ and $M_{S}$ with a reasonable accuracy from the $p_{T}$ distribution.

For the signal (3), we should consider the subprocesses $e \gamma \rightarrow S \bar{u}$ and $e g \rightarrow S \bar{u}$. In the former case there are contributions of the electron in the s-channel, $u$-quark in the t-channel, and of the $S$ in the u-channel, leading to

$$
\left(\frac{d \hat{\sigma}}{d \hat{t}}\right)_{e \gamma \rightarrow S \bar{u}}=\frac{\lambda^{2} \alpha}{72} \frac{1}{(-\hat{s} \hat{t})}\left\{\left[2+\frac{\hat{u}}{\hat{u}-M_{S}^{2}}+\frac{3(\hat{u}+\hat{t})}{\hat{s}}\right]^{2}+\left(\frac{\hat{u}}{\hat{u}-M_{S}^{2}}+\frac{3 \hat{u}}{\hat{s}}\right)^{2}\right\} .
$$

On the other hand, the cross section for $e g \rightarrow S \bar{u}$ receives contribution from the $u$-quark in the t-channel and from $S$ in the u-channel, giving rise to

$$
\left(\frac{d \hat{\sigma}}{d \hat{t}}\right)_{e g \rightarrow S \bar{u}}=\frac{\lambda^{2} \alpha_{s}}{16} \frac{1}{(-\hat{s} \hat{t})}\left[\frac{\hat{u}^{2}+M_{S}^{4}}{\left(\hat{u}-M_{S}^{2}\right)^{2}}\right]
$$

Figure 5 exhibits the total cross section, as a function of $M_{S}$, of the process $e \gamma \rightarrow S+$ jet taking into account the subprocesses (17) and (18), for $\sqrt{s}=500,1000$, and $2000 \mathrm{GeV}$. In order to regulate the co-linear divergences associated to the $t$-channel, we required that the angle $(\theta)$ of the produced jet, or of the leptoquark, with the beam pipe satisfies $|\cos \theta|<0.9$. It is interesting to notice that this cross section is dominated, for small $M_{S}$, by the gluon initiated process, while the direct photon contribution dominates for large $M_{S}$.

Once the leptoquark couples to $e u$ and $\nu d$ pairs with the same strength, the signal for its production in association with a jet is either 2 jets $+e$ or 2 jets $+\mathrm{E}_{\text {missing. }}$. The main backgrounds for these signals come from the reactions $e \gamma \rightarrow Z e$, and $e \gamma \rightarrow W \nu$ with the $W$ and $Z$ decaying into two jets [20]. We can overcome this background by requiring that the invariant mass of the jet pair is not close to $M_{W, Z}$. Another potential background is 
the Bethe-Heitler production of hadrons $(\gamma e \rightarrow q \bar{q} e)$, which possesses a large cross section. However, the main contribution to this processes comes from particles produced with small momenta. This allows us to reject with high efficiency this class of events by demanding that the observed particles and jets have a sufficiently high $p_{T}$.

In order to estimate the capability of the planned $e \gamma$ colliders to search for leptoquarks, we present in Fig. 6 the discovery contour in the $\left(\lambda \times M_{S}\right)$ plane. For the process (11), we required the significance level of the signal to be $5 \sigma$, when we consider a bin of $\pm 10 \mathrm{GeV}$ around $M_{S}$. We can see from Fig. 6 that we can observe leptoquarks with masses up to the kinematical limit of the machine provided that the coupling $\lambda$ is larger than $\sim 5 \times 10^{-2}$.

In Fig. 6] we also present the discovery contour for single leptoquark production (3), assuming a luminosity of $10^{4} \mathrm{pb}^{-1}$ per year for the $e \gamma$ collider. We required in this case the occurence of 25 events per year. The maximum value of leptoquark mass that can be reached is $M_{S} \simeq 850 \mathrm{GeV}$ for a center-of-mass energy of the $e^{+} e^{-}$machine of $1000 \mathrm{GeV}$, taking $\lambda=0.15$. Therefore, even for small leptoquark coupling, an $e \gamma$ collider can investigate a leptoquark almost up to the kinematical limit.

\section{ACKNOWLEDGMENTS}

This work was partially supported by Conselho Nacional de Desenvolvimento Científico e Tecnológico (CNPq), and Fundação de Amparo à Pesquisa do Estado de São Paulo (FAPESP). 


\section{REFERENCES}

[1] L. Abbott and E. Farhi, Phys. Lett. 101B (1981) 69; Nucl. Phys. B189 (1981) 547.

[2] S. Dimopoulos, Nucl. Phys. B168 (1981) 69 ; E. Farhi and L. Susskind, Phys. Rev. D20 (1979) 3404; J. Ellis et. al., Nucl. Phys. B182 (1981) 529.

[3] See, for instance, P. Langacker, Phys. Rep. 72 (1981) 185.

[4] J. L. Hewett and T. G. Rizzo, Phys. Rep. 183 (1989) 193.

[5] J. Wudka, Phys. Lett. 167B (1986) 337.

[6] J. F. Gunion and E. Ma, Phys. Lett. 195B (1987) 257; A. Dobado, M. J. Herrero and C. Munoz, Phys. Lett. 191B (1987) 449.

[7] W. Buchmüller, R. Rückl, and D. Wyler, Phys. Lett. 191B (1987) 442.

[8] O. J. P. Éboli and A. V. Olinto, Phys. Rev. D38 (1988) 3461; A. Dobado, M. J. Herrero and C. Munoz, Phys. Lett. 207B (1988) 97; J. L. Hewett and S. Pakvasa, Phys. Rev. D37 (1988) 3165; M. de Montigny and L. Marleau, Phys. Rev. D41 (1990) 3523.

[9] J. L. Hewett and T. G. Rizzo, Phys. Rev. D36, (1987) 3367; O. J. P. Éboli and J. E. Cieza-Montalvo, Phys. Rev. D47 (1993) 837; J. Blumlein and R. Ruckl, Phys. Lett. 304B (1993) 337.

[10] J. L. Hewett and S. Pakvasa, Phys. Lett. 227B (1989) 178; H. Nadeau and D. London, Univ. Montreal Report, UdeM-LPN-TH-132 (1993).

[11] F. R. Arutyunian and V. A. Tumanian, Phys. Lett. 4 (1963) 176; R. H. Milburn, Phys. Rev. Lett. 10 (1963) 75. 
[12] For a review see, V. Telnov Nucl. Instr. Meth. A294 (1990) 72.

[13] W. Buchmüller and D. Wyler, Phys. Lett. 177B (1986) 377.

[14] M. S. Gold (CDF Collab.), talk given at the XXVI International Conference in High Energy Physics, Dallas (1992).

[15] E. Witten, Nucl. Phys. B120 (1977) 189.

[16] D. W. Duke and J. F. Owens, Phys. Rev. D26 (1982) 1600.

[17] M. Drees and K. Grassie, Z Phys. C28 (1985) 451.

[18] H. Abramowicz, K. Charchula, and A. Levy, Phys. Lett. B269 (1991) 458.

[19] This cross section has been evaluated in Ref. [5]. We reproduce it here, fixing a minor misprint.

[20] The background associated to bremsstrahlung photons has been analyzed by E. Yehudai, Phys. Rev. D42 (1990) 771. 


\section{FIGURES}

FIG. 1. Invariant mass distribution for the process $e^{+} e^{-} \rightarrow e+q_{\gamma} \rightarrow e+$ jet. We considered an $e^{+} e^{-}$collider with $\sqrt{s}=500 \mathrm{GeV}$ and fixed $M_{S}=250 \mathrm{GeV}$. The curves are for the leptoquark coupling $\lambda=e$ (solid line), and $\lambda=1.0$ (dashed line). The dotted line represents the Standard Model prediction i.e. $\lambda=0$.

FIG. 2. The same as Figure 1 for $\sqrt{s}=1000 \mathrm{GeV}$.

FIG. 3. Transverse momentum distribution of the quark (jet) for the $e^{+} e^{-} \rightarrow e+q_{\gamma} \rightarrow$ jet $+\mathrm{E}_{\text {missing. }}$. We considered an $e^{+} e^{-}$collider with $\sqrt{s}=500$ $\mathrm{GeV}$ and fixed $M_{S}=250 \mathrm{GeV}$. The curves are for the leptoquark coupling $\lambda=e$ (solid line), and $\lambda=1.0$ (dashed line). The dotted line represents the Standard Model prediction i.e. $\lambda=0$.

FIG. 4. The same as Figure 3 for $\sqrt{s}=1000 \mathrm{GeV}$.

FIG. 5. Total cross section for the process $e^{+} e^{-} \rightarrow e+\gamma\left(g_{\gamma}\right) \rightarrow S+$ jet as a function of $M_{S}$ with $\lambda=e$ and $\sqrt{s}=500 \mathrm{GeV}$ (dotted line), $\sqrt{s}=1000 \mathrm{GeV}$ (solid line), and $\sqrt{s}=2000 \mathrm{GeV}$ (dashed line). We added the contributions from the subprocesses (17) and (18)

FIG. 6. Discovery contour in the plane $\left(\lambda \times M_{S}\right)$ : (a) for the resonant production $e+q_{\gamma} \rightarrow S \rightarrow e+$ jet (lower cuves); (b) for single leptoquark production $e^{+} e^{-} \rightarrow e+\gamma\left(g_{\gamma}\right) \rightarrow S+$ jet (upper curves). The curves represent an $e^{+} e^{-}$collider with $\sqrt{s}=500 \mathrm{GeV}$ (dotted line), $\sqrt{s}=1000$ $\mathrm{GeV}$ (solid line), and $\sqrt{s}=2000 \mathrm{GeV}$ (dashed line). We assumed a luminosity of $10^{4} \mathrm{pb}^{-1}$ per year. 
Figure 1

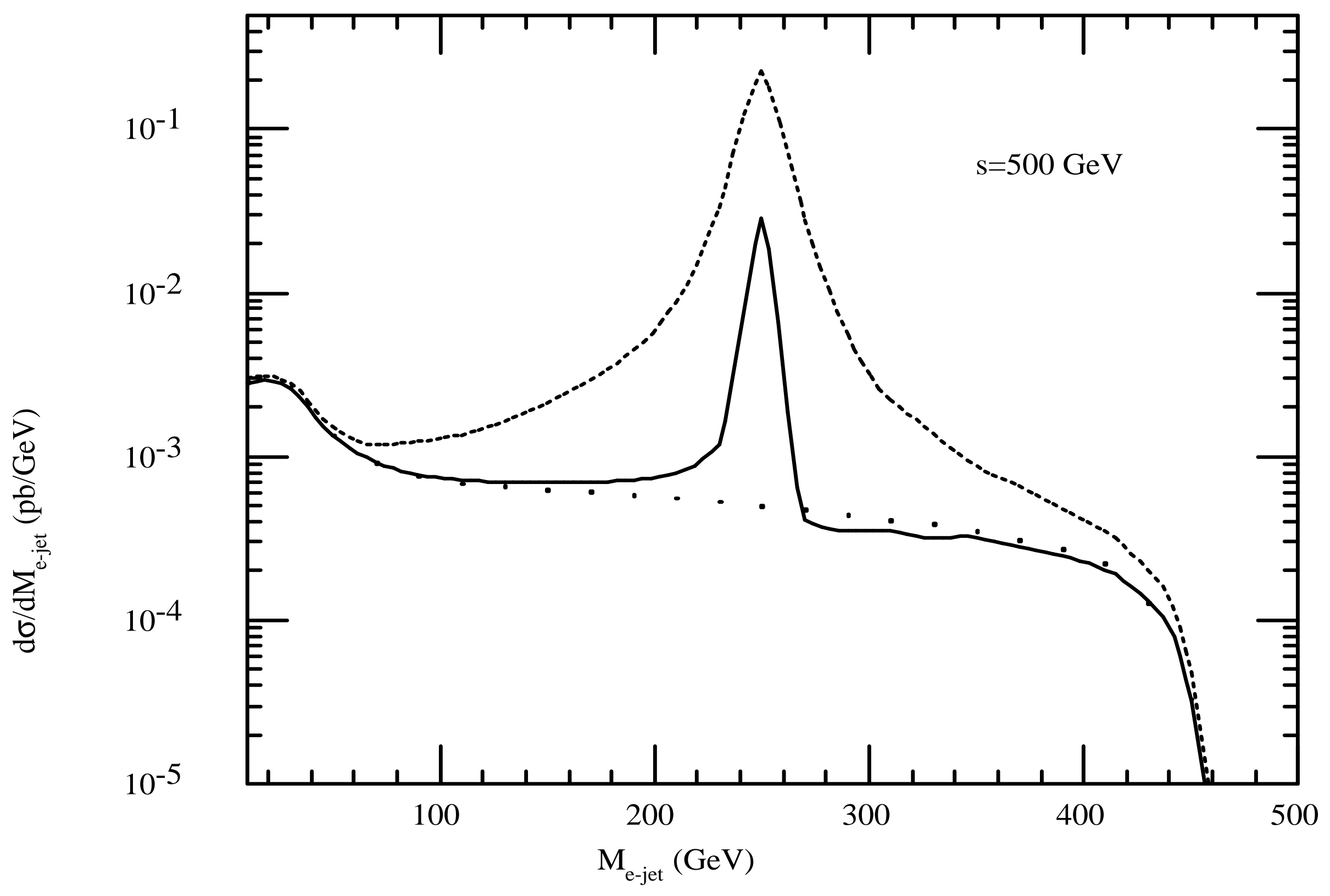


Figure 2

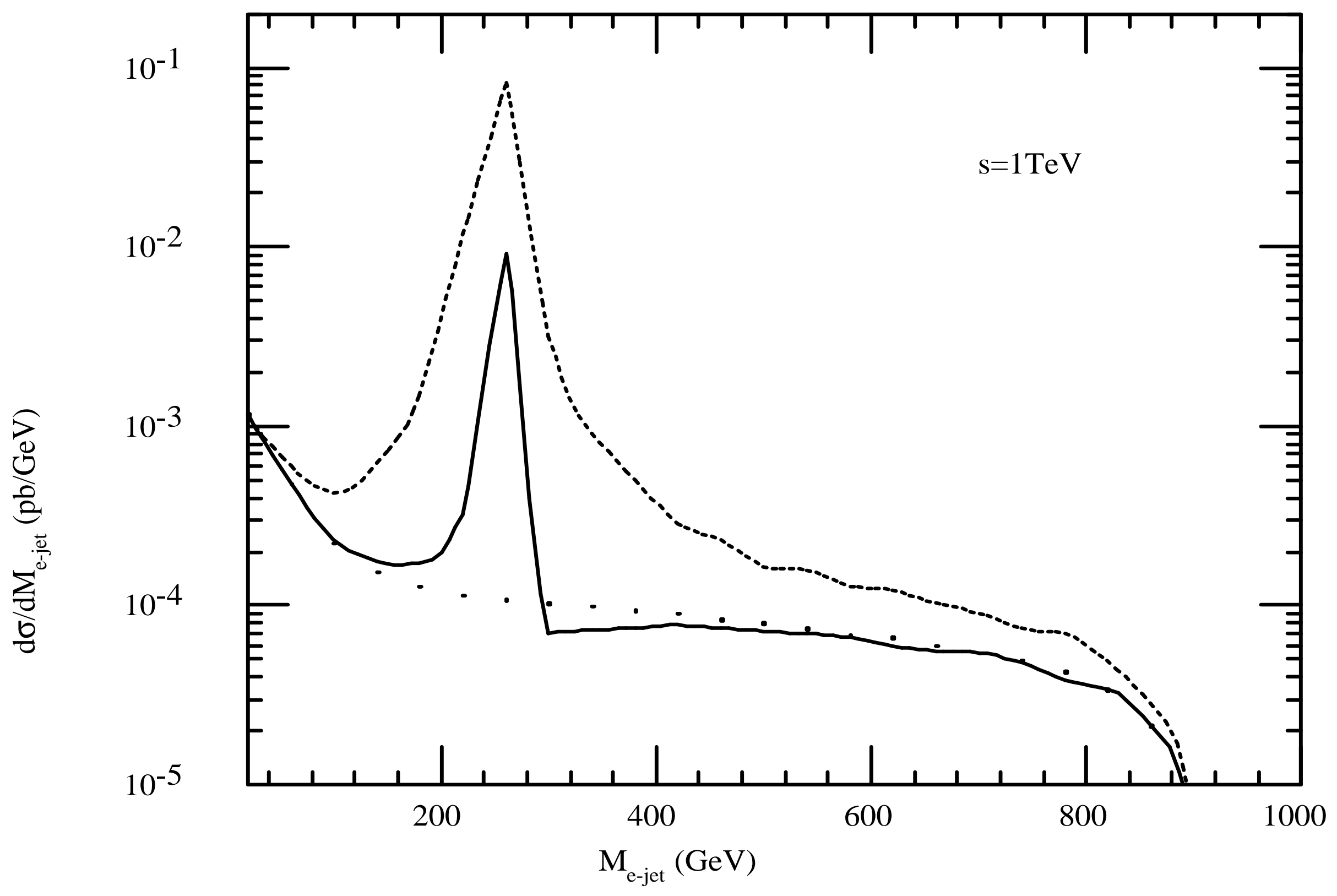


Figure 3

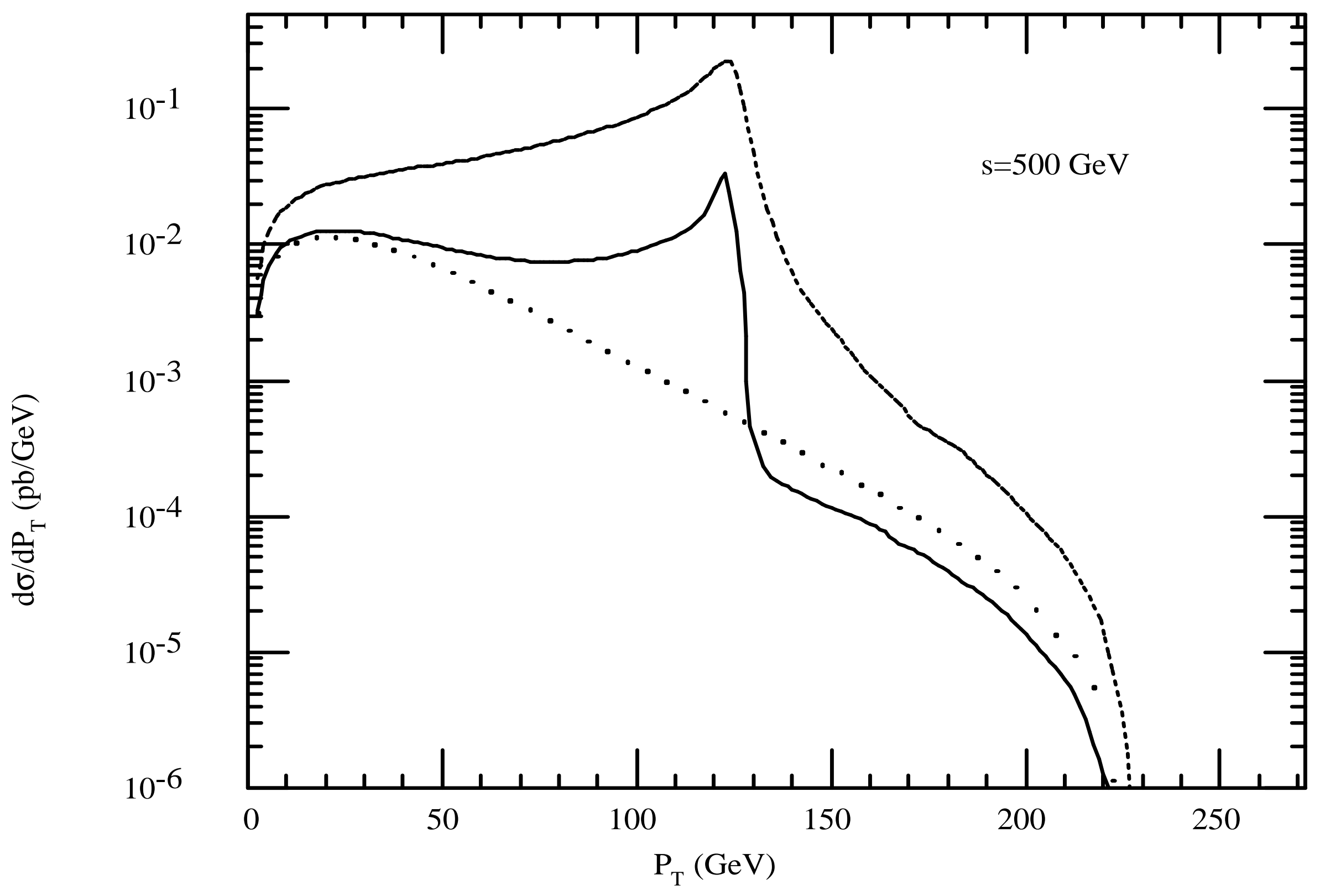


Figure 4

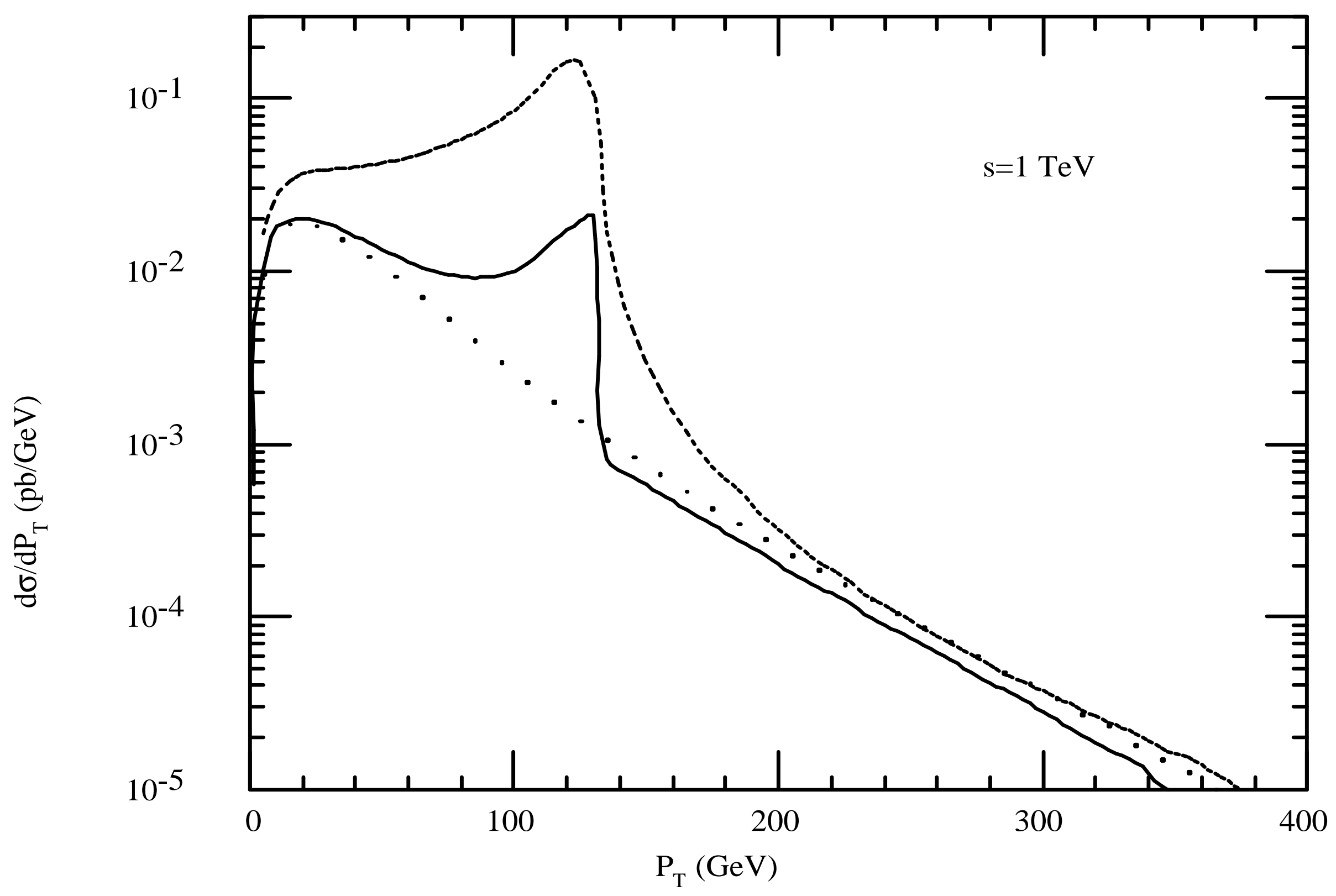


Figure 5

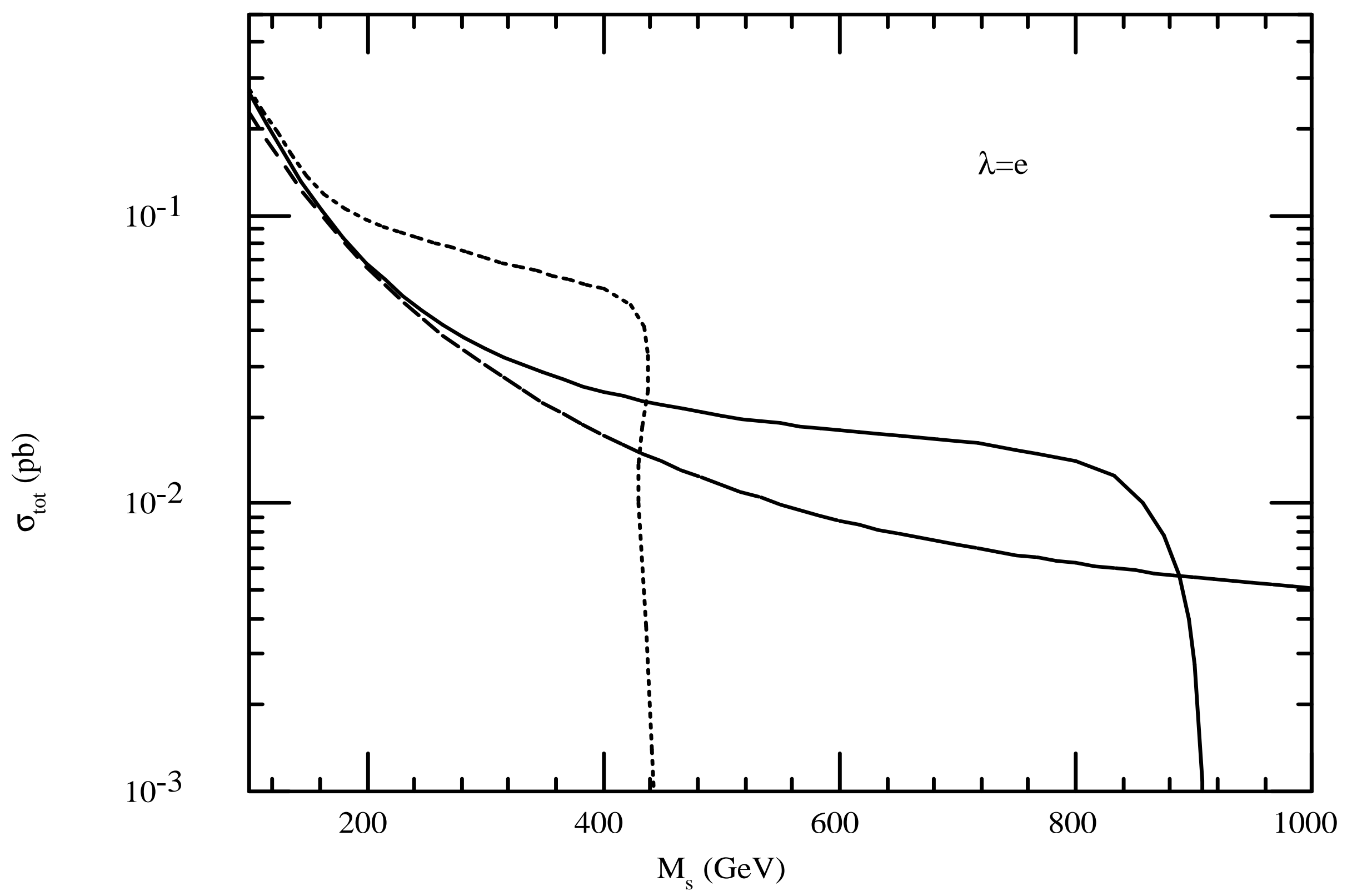


Figure 6

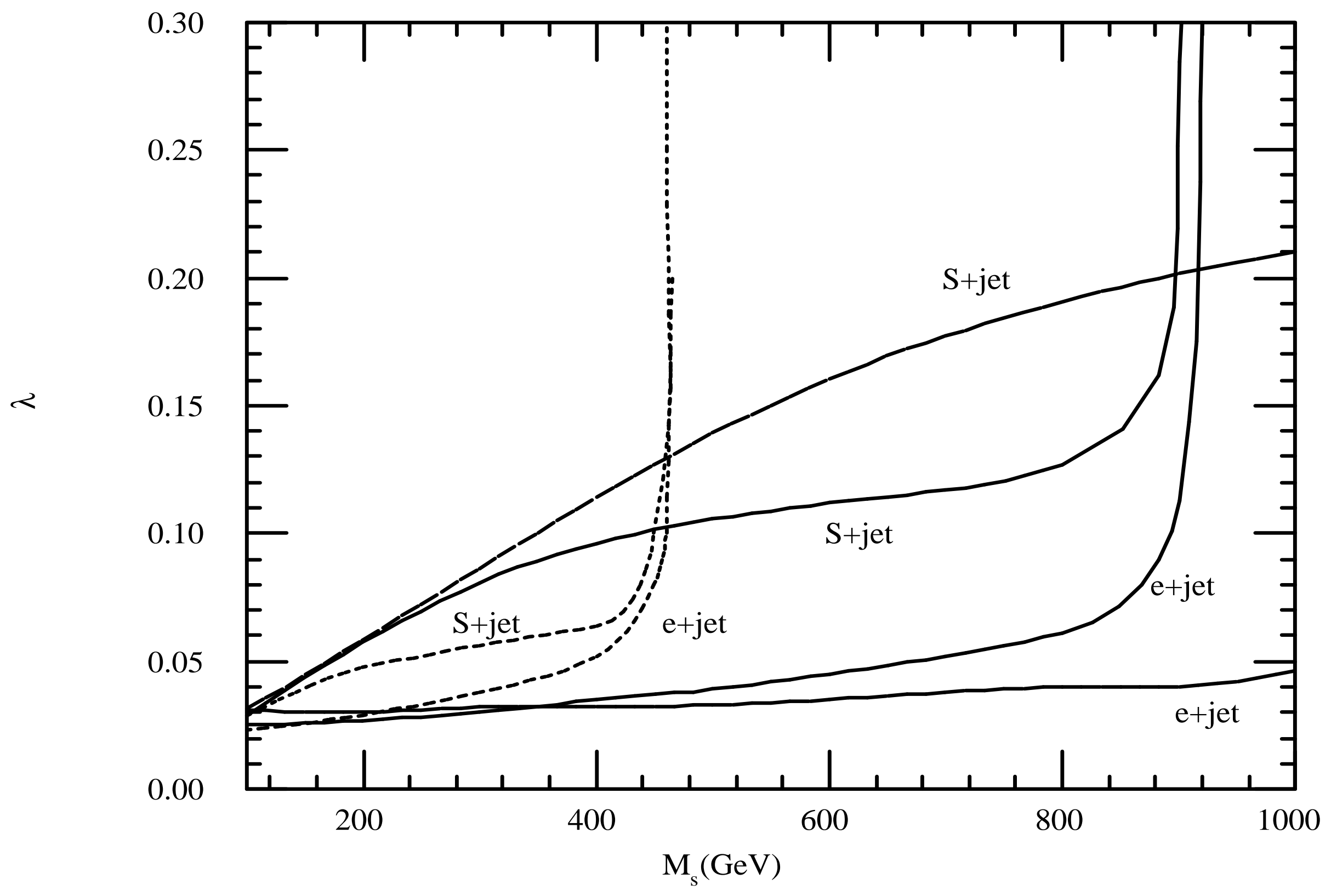

